\title{
Preparation and investigation of hydrolyzed polyacrylonitrile as a preliminary biomedical hydrogel
}

\author{
Ji Hoon Park ${ }^{1 \dagger}$, Guo Zhe Tai ${ }^{1 \dagger}$, Bo Keun Lee ${ }^{1}$, Seung Hun Park', Ja Yong Jang ${ }^{1}$, Jung Soo Lee ${ }^{1,2}$, Jae Ho Kim', \\ Kwideok Park ${ }^{3}$, Ju Woong Jang ${ }^{2}$ and Moon Suk Kim ${ }^{1 *}$
}

\begin{abstract}
Background: Hydrolyzed polyacrylonitrile (HPAN) has attracted much attention as a hydrogel for a broad range of biomedical applications. Therefore, in this study, we prepared HPAN derivatives with controllable compositions by the radical polymerization of acrylonitrile (AN), methacrylic acid (MAA) and N-isopropylacrylamide (NIPAM) monomers.

Results: The prepared poly(AN-co-MAA-CO-NIPAM) copolymers had different ratios of AN, MAA, and NIPAM and molecular weights ranging from 2000 to 50,000. The copolymers were prepared as films to examine their properties. The prepared copolymer films showed different solubilities, contact angles, and swelling ratios. The properties of the copolymer films were affected by the hydrophobic PAN segments and the hydrophilic PMAA or PNIPAM segments.

Conclusion: Thus, we conclude that introducing PMAA and PNIPAM segments with different ratios and lengths into PAN segments could represent a method of controlling the hydrogel properties of copolymers.
\end{abstract}

Keywords: Hydrolyzed polyacrylonitrile, Radical polymerization, Swelling, Hydrogel, Biomedical applications

\section{Background}

Hydrogels have been developed extensively for a broad range of biomedical applications [1, 2]. Most types of hydrogels can absorb large quantities of water relative to their initial weight because of their intrinsic hydrophilicity. Because of this propensity to retain large amounts of water, hydrogels can be used in biomedical applications in dehydrated and/or hydrated form. Hydrogels have been applied extensively as smart polymers for drug carriers, contact lens materials, and orthopedic implants [3-7]. In vivo, hydrogels expand to form swelled shapes by absorbing body-derived fluids [8-11].

Many hydrogels have been developed using various polymers. Among them, partial hydrolyzed polyacrylonitrile (HPAN) is produced through a chemical reaction of polyacrylonitrile (PAN) with sodium hydroxide [12-14]. The reaction produces a water-soluble HPAN block

\footnotetext{
* Correspondence: moonskim@ajou.ac.kr

Ji Hoon Park and Guo Zhe Tai are equal first authors

${ }^{\dagger}$ Equal contributors

'Department of Molecular Science and Technology, Ajou University, Suwon 443-759, South Korea

Full list of author information is available at the end of the article
}

copolymer consisting of hydrophobic nitrile functional groups and hydrophilic poly(acrylic acid), partially neutralized poly(acrylic acid), and poly(acrylamide) [15-18].

HPAN mainly expands in water and/or in body-derived fluids to form swollen hydrogels that are highly biocompatible and biodurable and cause minimal inflammation following implantation. Uniquely, HPAN shows elasticity and tensile strength that are very similar to those of body tissues, such as cartilage and the nucleus pulposus of the intervertebral disc [19-21]. Thus, HPAN has been developed extensively for minimally invasive spine surgery [22].

Although HPAN has a significant advantage in biomedical applications, control over the hydrophilic and hydrophobic segments is limited by the hetero-chemical reaction of PAN with sodium hydroxide and/or amine. Thus, developing a simple preparation method for HPAN copolymers with controllable composition has been the subject of practical development efforts.

HPAN derivatives can be easily prepared using acrylonitrile (AN), methacrylic acid (MAA) and $N$ isopropylacrylamide (NIPAM) monomers, which are cheap and easy to polymerize. The prepared poly(AN-Co-MAA- 
Table 1 Preparation and swelling ratios of poly(AN-CO-MAA-CO-NIPAM), poly(AN-CO-MAA) and poly(AN-co-NIPAM) copolymers

\begin{tabular}{lllllll}
\hline & Molar ratio (\%) (AN-MAA-NIPAM) & Molar ratio (\%) (AN-MAA-NIPAM) & $M_{w}$ & Yield (\%) & Swelling ratio (\%) & Solubility (mg/mL) \\
\hline P(AN-MAA-NIPAM) & $50: 25: 25$ & $47: 23: 30$ & 2000 & 89 & 65 & 12 \\
& $25: 50: 25$ & $25: 52: 23$ & 2000 & 61 & 72 & 14 \\
& $25: 25: 50$ & $22: 24: 54$ & 2000 & 90 & 81 & 21 \\
& $50: 25: 25$ & $49: 26: 25$ & 20000 & 82 & 62 & 5 \\
P(AN-MAA) & $50: 25: 25$ & $47: 24: 29$ & 5000062 & 58 & $\sim 1$ \\
P(AN-NIPAM) & $50: 50: 0$ & $53: 47: 0$ & 2000 & 93 & 60 & 10 \\
\hline
\end{tabular}

${ }^{\mathrm{a}}$ Determined by ${ }^{13} \mathrm{C}-\mathrm{NMR}$

co-NIPAM) copolymer derivatives have the ability to absorb higher water and form hydrogel, which is an essential criterion for a hydrogel suitable for biomedical applications.

To the best of our knowledge, few previous studies have addressed the preparation of HPAN derivatives with controllable compositions. Thus, in this work, we prepared HPAN derivatives with controllable compositions of AN, MAA and NIPAM by radical polymerization. The solution and swelling properties of the copolymer were also examined for hydrogel application.

\section{Methods}

\section{Materials}

Acrylonitrile (AN), methacrylic acid (MAA), and $N$-isopropylacrylamide (NIPAM) were purchased from Aldrich (MO, USA) and distilled over $\mathrm{CaH}_{2}$ under reduced pressure. Azobisisobutyronitrile (AIBN) was recrystallized in methanol. THF was purchased from Burdick \& Jackson (MI, USA). The HPLC-grade $n$-hexane and ethyl ether were purchased from Samchun (Pyeongtaek, Korea) and used as received.

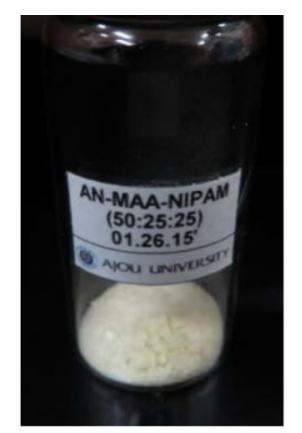

AN-MAA-NIPAM (50:25:25) $2 \mathrm{k}$

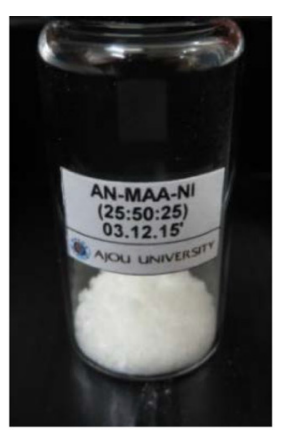

AN-MAA-NIPAM $(25: 50: 25) 2 \mathrm{k}$

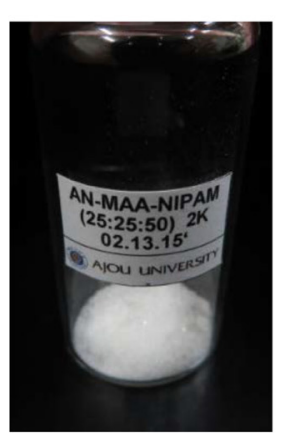

AN-MAA-NIPAM

(25:25:50) 2k

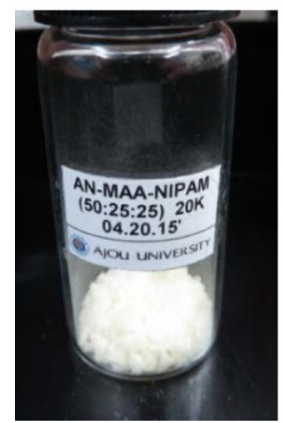

AN-MAA-NIPAM AN-MAA-NIPAM (50:25:25) 20k

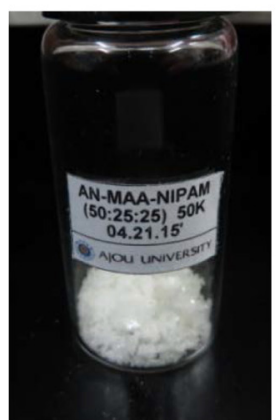

(50:25:25) 50k

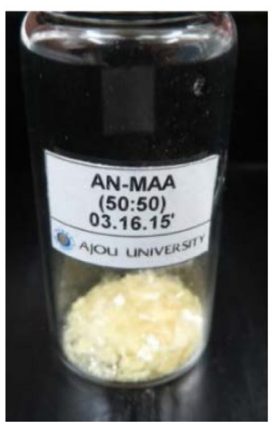

AN-MAA $(50: 50) 2 k$

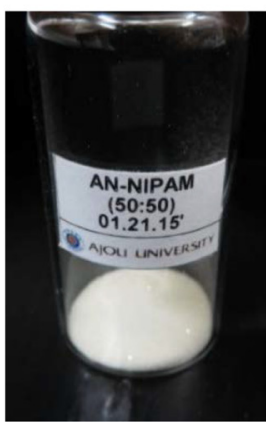

AN-NIPAM (50:50) $2 \mathrm{k}$

Fig. 1 Scheme of polymers. Scheme of the polymerization of poly(AN-co-MAA-co-NIPAM) and pictures of the obtained poly(AN-co-MAA-coNIPAM), poly(AN-co-MAA) and poly(AN-Co-NIPAM) copolymers 


\section{Synthesis of poly(AN-co-MAA-co-NIPAM)}

All glassware was dried by heating in vacuum and handled under a dry nitrogen stream. The typical polymerization process to produce poly(AN-co-MAAco-NIPAM) with a AN/MAA/NIPAM ratio of 50/25/25 and a molecular weight of 2000 is as follows: AIBN $(0.43 \mathrm{~g}, 2.6 \mathrm{mmol})$ and THF $(40 \mathrm{~mL})$ were introduced into a flask. Then, AN (1.39 g, $26.2 \mathrm{mmol})$, MAA (1.13 g, $13.1 \mathrm{mmol})$, and NIPAM (1.48 g, $13.1 \mathrm{mmol})$ were added by syringe and bubbled with dry nitrogen for $30 \mathrm{~min}$ to remove oxygen from the reaction solution. The solution was stirred vigorously at $75^{\circ} \mathrm{C}$ under a dry nitrogen atmosphere. After stirring for $16 \mathrm{~h}$, the reaction mixture was poured into ethyl ether to precipitate the copolymer, which was separated from the supernatant by decantation and then filtered. The resulting polymer was dried under vacuum to yield the copolymers. The copolymers are summarized in according to their feed compositions Table $1 .{ }^{1} \mathrm{H}$-NMR and ${ }^{13} \mathrm{C}$-NMR spectra were measured using a Varian Mercury Plus 400 system with DMSO- $d_{6}$ in the presence of tetramethylsilane. The AN/ MAA/NIPAM ratios in the poly(AN-co-MAA-coNIPAM) copolymers were determined from the ${ }^{13} \mathrm{C}$ NMR spectra by comparing the average integration value of the characteristic carbonyl signal of AN/MAA/ NIPAM.

\section{Synthesis of poly(AN-co-MAA) and poly(AN-co-NIPAM)} Poly(AN-co-MAA) with an AN/MAA ratio of 50/50 and poly(AN-co-NIPAM) with an AN/NIPAM ratio of
50/50 were prepared using same copolymerization method as described in the previous section.

\section{Determination of solution properties}

$250 \mathrm{mg}$ of the poly(AN-co-MAA-co-NIPAM), poly(ANco-MAA) and poly(AN-co-NIPAM) copolymers were introduced into 5-mL vials. Two milliliter of distilled water ( $\mathrm{pH}$ 7), a solution of $\mathrm{pH} 3$, and a solution of $\mathrm{pH} 10$ (Adjusted to the desire $\mathrm{pH}$ with $1 \mathrm{~N} \mathrm{HCl}$ and $\mathrm{NaOH}$ ) were added to the vials and incubated for $1 \mathrm{~h}$ and observed. The solubility of poly(AN-co-MAA-co-NIPAM), poly(AN-co-MAA) and poly(AN-co-NIPAM) copolymers in DW was determined at $37^{\circ} \mathrm{C}$.

\section{Preparation of copolymer films}

Poly(AN-co-MAA-co-NIPAM), poly(AN-co-MAA) and poly(AN-co-NIPAM) films were prepared using solvent casting. One milligram of copolymers was solubilized in $1 \mathrm{~mL}$ of THF. The solution was casted on polyethylene film and allowed to dry slowly at $10{ }^{\circ} \mathrm{C}$ for $2 \mathrm{~d}$. Next, the casted films were dried in a vacuum oven at room temperature for 4 days, resulting in smooth and nonporous films. The copolymer films were cut into discs with $6 \mathrm{~mm}$ diameters and $200 \mu \mathrm{m}$ thicknesses.

\section{Contact angle measurement of copolymer films}

The water contact angle was measured using the sessile drop method at room temperature with an optical benchtype contact angle goniometer (Phoenix 150, SEO, Suwon, Korea). One drop of purified water $(5 \mu \mathrm{L})$ was deposited

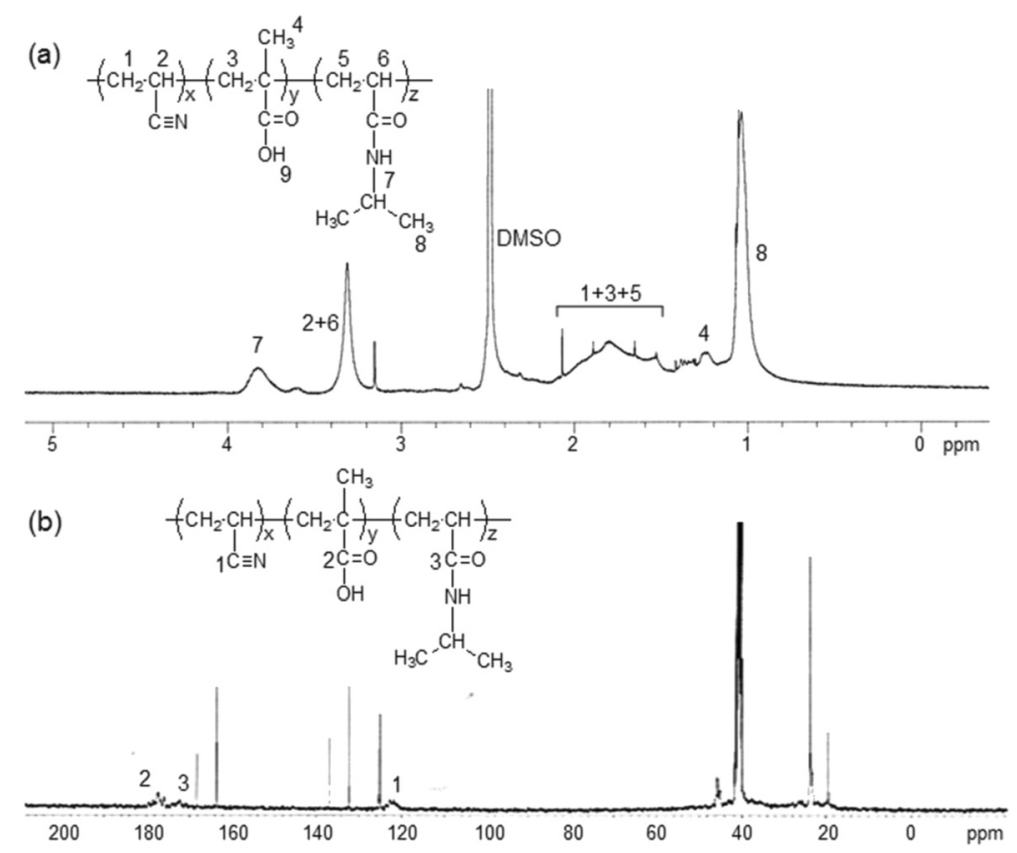

Fig. 2 NMR spectra of polymer. a ${ }^{1} \mathrm{H}-\mathrm{NMR}$ and $\mathbf{b}{ }^{13} \mathrm{C}-\mathrm{NMR}$ spectra of poly(AN-CO-MAA-CO-NIPAM) with a ratio of 50/25/25 in DMSO- $d_{6}$ 
onto the prepared film surface by means of a microsyringe. The water contact angle was measured within $5 \mathrm{~s}$.

\section{Determination of swelling ratios}

The completely dried poly(AN-co-MAA-co-NIPAM), poly(AN-co-MAA) and poly(AN-co-NIPAM) films were placed in $5-\mathrm{mL}$ vials, and $1 \mathrm{~mL}$ of $\mathrm{PBS}$ at $37{ }^{\circ} \mathrm{C}$ was added. The swollen films were removed after $24 \mathrm{~h}$, and the surface was quickly blotted free of water with filter paper. The films were then weighed and placed in the same bath. The mass measurements were continued until equilibrium was reached. The equilibrium swelling ratio was determined according to the conventional gravimetric method using the following equation: Swelling ratio $(\%)=$ [equilibrium swollen weight - initial dried weight) $\times 100] /$ [initial dried weight] .

\section{Results and discussion}

Preparation of poly(AN-co-MAA-co-NIPAM), poly(AN-coMAA) and poly(AN-co-NIPAM)

The preparation of poly(AN-co-MAA-co-NIPAM), poly(AN-co-MAA) and poly(AN-co-NIPAM) copolymers is summarized in Table 1. All copolymers were synthesized through the radical polymerization of the monomers AN, MAA, and NIPAM using the AIBM as an initiator. All copolymers were obtained as colorless or slight yellowish copolymers after isolation by precipitation (Fig. 1). The poly(AN-co-MAA-co-NIPAM) copolymers were prepared with molecular weights ranging from 2000 to 50,000 using different ratios of $\mathrm{AN}$, MAA, and NIPAM.

Figure 2 shows the ${ }^{1} \mathrm{H}-\mathrm{NMR}$ and ${ }^{13} \mathrm{C}$-NMR spectra of poly(AN-co-MAA-co-NIPAM). Poly(AN-co-MAA-coNIPAM) copolymer exhibited characteristic peaks of PAN, PMAA, and PNIPAM. The methyl, methylene and methine protons were observed in ${ }^{1} \mathrm{H}$-NMR spectra. The carbons of the carbonyl groups in PAN, PMAA, and PNIPAM were observed at $\delta=122,177$, and $172 \mathrm{ppm}$ in ${ }^{13} \mathrm{C}$-NMR spectra. The ratio of PAN, PMAA, and PNIPAM was determined according to the carbon integration ratios of the carbonyl groups, which agreed well with the feed ratio values. The poly(AN-co-MAA) and poly(AN-co-NIPAM) also showed characteristic peaks of PAN, PMAA, or PNIPAM, and the ratio of the prepared (a)

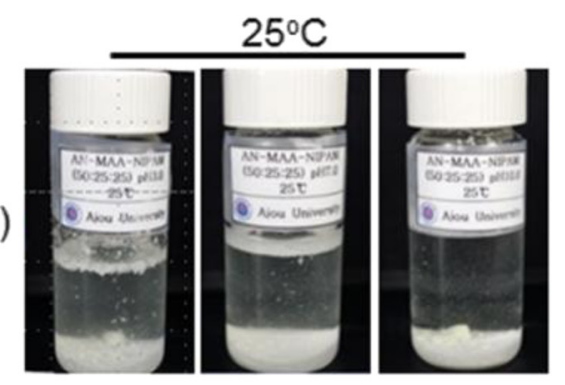

$\mathrm{pH} 3.0$

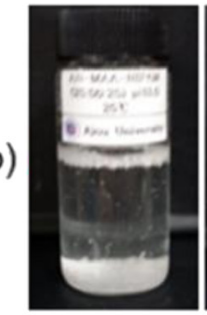

$\mathrm{pH} 3.0$

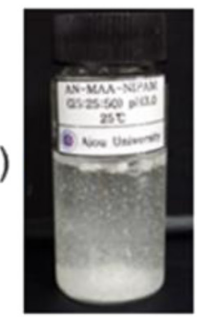

$\mathrm{pH} 3.0$

\begin{abstract}
$\mathrm{pH} 7.0$
\end{abstract}

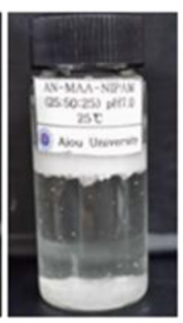

$\mathrm{pH} 7.0$



$\mathrm{pH} 7.0$
$\mathrm{pH} 10$

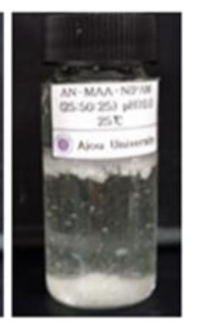

$\mathrm{pH} 10$

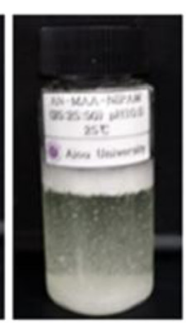

$\mathrm{pH} 10$
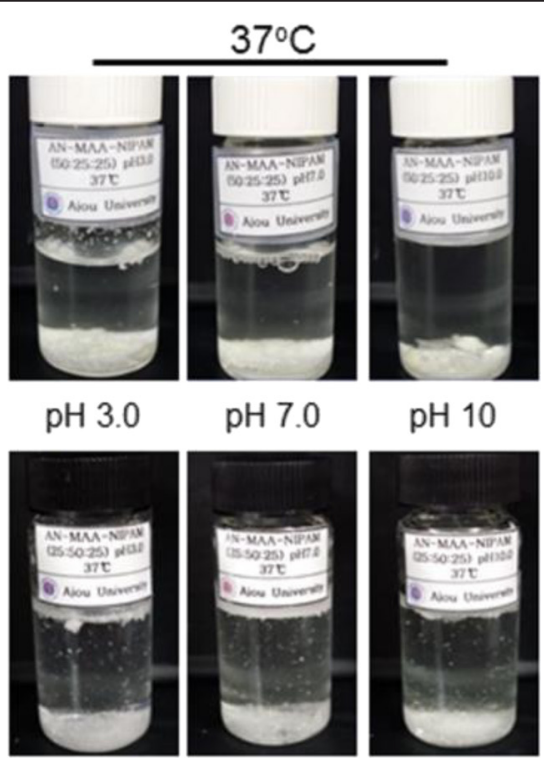

pH 3.0

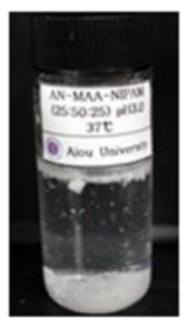

pH 3.0
$\mathrm{pH} 7.0$

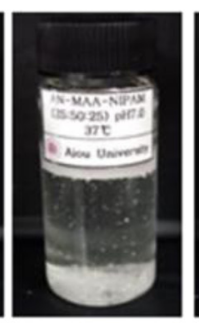

pH 7.0



$\mathrm{pH} 7.0$
$\mathrm{pH} 10$

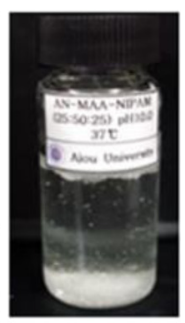

pH 10

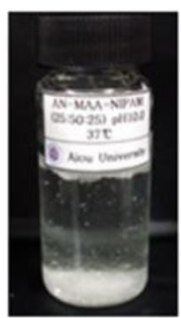

pH 10 (d)

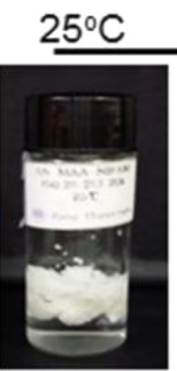

$37^{\circ} \mathrm{C}$



(e)


(f)
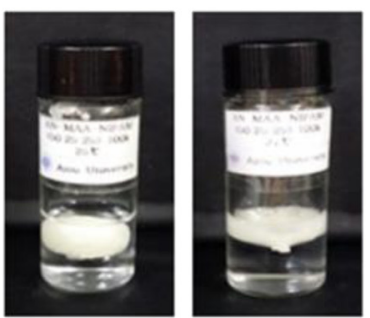

Fig. 3 Pictures of polymer. Pictures of poly(AN-Co-MAA-CO-NIPAM), poly(AN-co-MAA) and poly(AN-co-NIPAM) copolymer solutions with ratios of (a) 50/25/25, (b) 25:50:25, and (c) 25:25:50 (AN:MAA:NIPAM) at pH 3.0, 7.0, and 10.0 and with molecular weights of (d) 2000, (e) 20,000 and f 50,000 

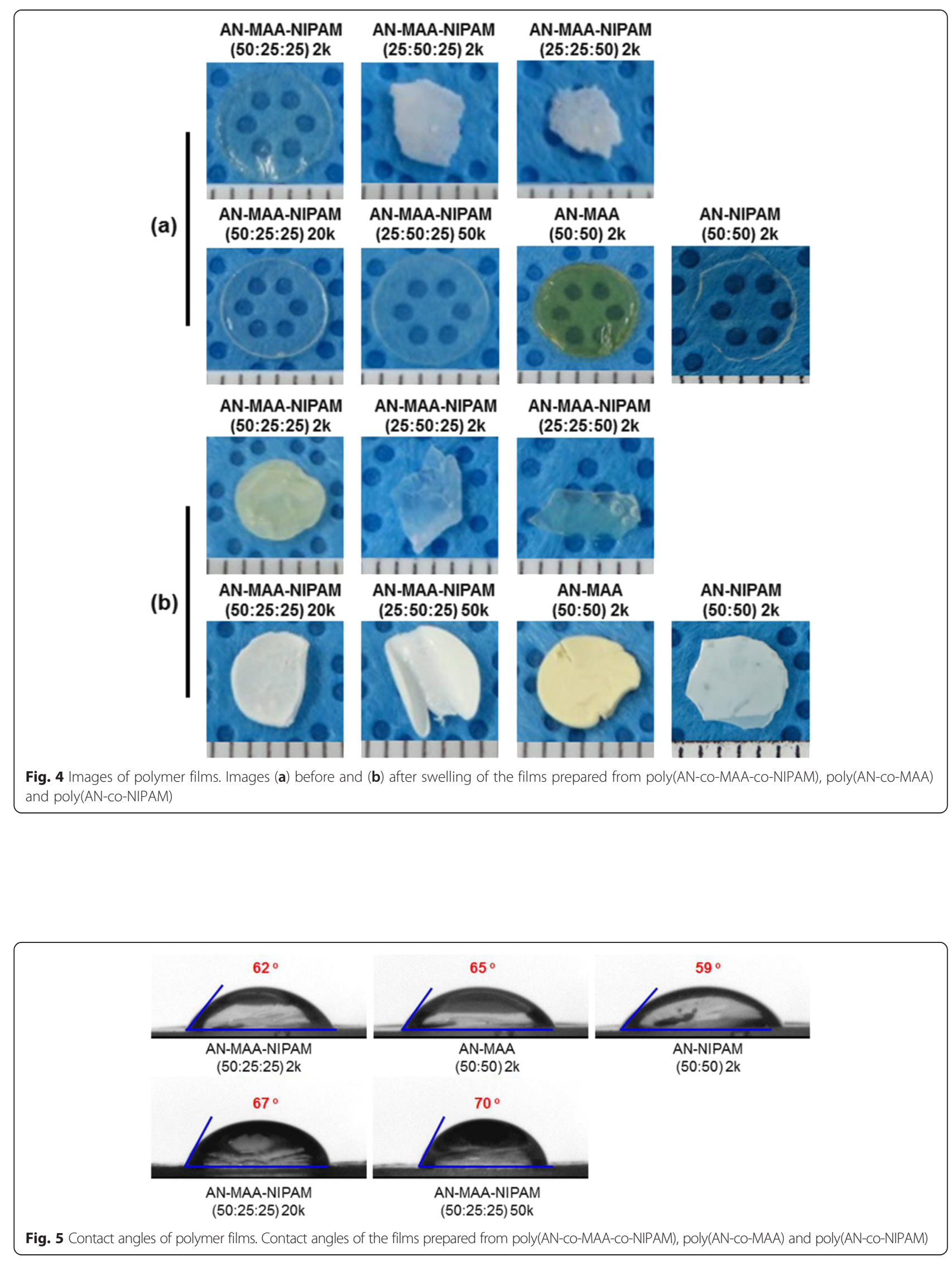


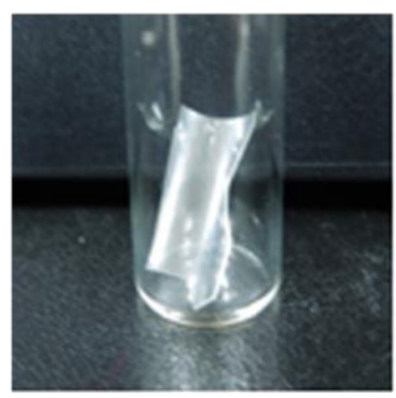

(a)

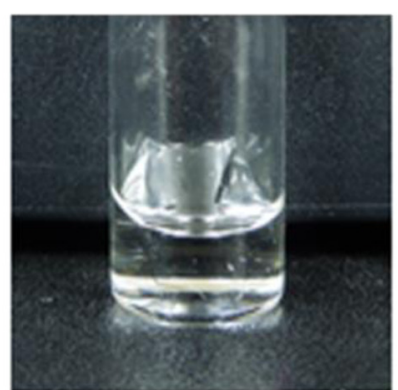

(b)

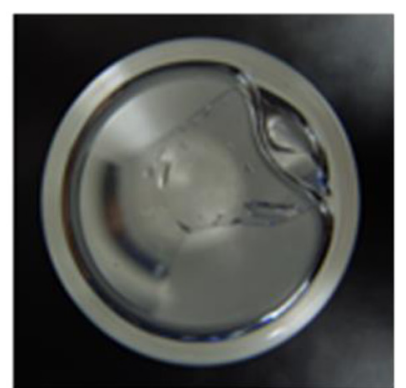

(c)

Fig. 6 Pictures of polymer films. Pictures of (a) poly(AN-CO-MAA-Co-NIPAM) film; (b) side and (c) top views in medium

copolymers determined by ${ }^{13} \mathrm{C}$-NMR was also in good agreement with the feed ratio values as summarized in Table 1.

These findings indicated that HPAN derivatives with controllable compositions of AN-MAA-NIPAM were successfully prepared by radical polymerization. Thus, in this work, we have demonstrated that it is possible to prepare copolymers with distinct compositions, lengths and molecular weights of the hydrophobic PAN segment and the hydrophilic PMAA and/or PNIPAM segments.

\section{Solution properties of poly(AN-co-MAA-co-NIPAM), poly(AN-Co-MAA) and poly(AN-Co-NIPAM)}

To examine the solution properties of poly(AN-co-MAAco-NIPAM), poly(AN-co-MAA) and poly(AN-co-NIPAM), the aqueous solutions of the copolymers were prepared by dissolving them in solutions of $\mathrm{pH} \mathrm{3,7}$, and 10. Figure 3 shows pictures of 10 -wt\% copolymers at $\mathrm{pH} 3,7$, and 10 at $25{ }^{\circ} \mathrm{C}$ and $37^{\circ} \mathrm{C}$. The copolymer solutions showed different solubility as summarized in Table 1 . The solubility of poly(AN-co-MAA-Co-NIPAM) with a ratio of 50/25/25 exhibited less solubility than those with ratios of $25 / 50 / 25$ and 20/25/50 in similar molecular weights, which indicated that the solubility decreased as the amount of hydrophobic PAN segment increased. In addition, poly(AN-co-MAAco-NIPAM) appeared to become nearly insoluble as the molecular weight increased from 2000 to 20,000 and 50,000. Furthermore, poly(AN-co-MAA-co-NIPAM) with a ratio of 20/25/50 exhibited solubility of $10 \sim 17 \mathrm{mg} / \mathrm{mL}$. These findings indicate that the hydrophilic PNIPAM segment mainly affects the solution properties.

\section{Surface properties of poly(AN-co-MAA-co-NIPAM),} poly(AN-co-MAA) and poly(AN-co-NIPAM)

Copolymer films were prepared to examine the surface properties of poly(AN-co-MAA-Co-NIPAM), poly(ANco-MAA) and poly(AN-co-NIPAM) (Fig. 4a). The poly(AN-co-MAA-co-NIPAM) and poly(AN-co-MAA)




films with larger ratios of PAN exhibited a slight yellowish color. The films with larger ratios of NIPAM exhibited brittle properties.

As shown in Fig. 5, the contact angles of poly(AN-coMAA-co-NIPAM) of 2000, 20,000 and 50,000 were 62, 67 , and $70^{\circ}$ respectively, indicating slight increasing of contact angles as molecular weight increased. Meanwhile poly(AN-co-MAA) exhibited slight increasing to angles of $65^{\circ}$ and poly(AN-co-NIPAM) slightly decreased to $59^{\circ}$.

\section{Swelling properties of poly(AN-co-MAA-co-NIPAM), poly(AN-Co-MAA) and poly(AN-Co-NIPAM)}

Figure 6 shows the images of swollen poly(AN-coMAA-co-NIPAM). The prepared films were swollen in media. The mass measurements of swollen films were performed to determine the swelling ratio (Fig. $4 \mathrm{~b}$ ). The swelling ratios of the copolymers were summarized in Table 1. The swelling ratio of poly(AN-co-MAA-coNIPAM) with a ratio of $25 / 25 / 50$ was higher than those of poly(AN-co-MAA-Co-NIPAM) with ratios of 50/25/ 50 and $25 / 50 / 25$, suggesting that the higher hydrophilicities of the copolymer are correlated with increased PNIPAM segment. The swelling ratio increased slightly as the molecular weight increased. Poly(AN-co-MAA) and poly(AN-co-NIPAM) showed lower swelling ratios than poly(AN-co-MAA-co-NIPAM) with a ratio of $25 / 25 / 50$ because of the higher PAN segment concentration, even though all the copolymers had the same hydrophilic PMAA or PNIPAM segment concentration.

The surface structure of the films was examined by SEM. Figure 7 shows morphologic SEM images of the surface before and after swelling. The surface before swelling seemed to be covered with thin fibers in irregular structure, but non-porous films. After selling the films changed to structures assembled into fibrils of several micrometers in thickness and maintained the nonporous surfaces.

\section{Conclusion}

In this work, we successfully prepared HPAN derivatives with controllable compositions. The properties of the copolymer films depended on the ratio and length of the hydrophobic PAN segment and the hydrophilic PMAA and/or PNIPAM segments. Although future studies will be needed to provide additional biological information associated with cellular studies including cytoxicity, cell growth and proliferation as well as animal experiments, we anticipate that the HPAN derivatives with controllable compositions developed in this study can be potentially used as biomedical hydrogels.

\section{Competing interests}

The authors declare that they have no competing interests.

\section{Authors' contribution}

JHP and GZT performed the whole process of experiments and drafted the manuscript. BKL helped to conduct the experiment. SHP carried out the swelling tests and JYJ conducted SEM observations. JSL and JWJ participated in the design of the study. JHK and KP helped to reviewed the manuscript. MSK conceivedin the design of experiments, carried out review and the manuscript as a corresponding author. All authors read and approved the final manuscript.

\section{Acknowledgements}

This study was supported by a grant from a Small and Medium Business Administration (S2087373) and Priority Research Centers Program (20100028294) through the National Research Foundation of Korea (NRF) grant funded by the Korea government (MSIP).

\section{Author details}

'Department of Molecular Science and Technology, Ajou University, Suwon 443-759, South Korea. ${ }^{2}$ R\&DB Center, Cellumed Co., Ltd., Seoul 153-803, South Korea. ${ }^{3}$ Center for Biomaterials, Korea Institute of Science and Technology, Seoul 136-791, South Korea.

Received: 1 September 2015 Accepted: 19 October 2015 Published online: 26 October 2015

\section{References}

1. Choi S, Choi W, Kim S, Lee S, Noh I, Kim CW. Purification and biocompatibility of fermented hyaluronic acid for its applications to biomaterials. Biomater Res. 2014;18:6.

2. Kim DY, Kwon DY, Kwon JS, Kim JH, Min BH, Kim MS. Injectable in situforming hydrogels for regenerative medicines. Polymer Review. 2015:55:407-52.

3. Kim DY, Kwon DY, Kwon JS, Kim JH, Min BH, Kim MS. Stimuli-responsive injectable in situ-forming hydrogels for regenerative medicines. Polymer Review. 2015;55:407-52.

4. Gupta KC, Haider A, Choi YR, Kang IK. Nanofibrous scaffolds in biomedical applications. Biomater Res. 2014;18:5.

5. Ionov L. 3D microfabrication using stimuli-responsive self-folding polymer films. Polym Rev. 2013;53:92-107.

6. Park JH, Kang HY, Kwon DY, Lee BK, Lee B, Jang JW, et al. Biodegradable poly(lactide-co-glycolide-co- $\varepsilon$-caprolactone) block copolymers - evaluation as drug carriers for a localized and sustained delivery system. J Mater Chem B. 2015;3:8143-53.

7. Wang ZG, Wan LS, Xu ZK. Surface engineerings of polyacrylonitrile-based asymmetric membranes towards biomedical applications: an overview. J Memb Sci. 2007;304:8-23.

8. Seo HW, Kim DY, Kwon DY, Kwon JS, Jin LM, Lee B, et al. Injectable intratumoral hydrogel as 5-fluorouracil drug depot. Biomaterials. 2013;34:2748-57.

9. Wang Z, Zhang Y, Zhang J, Huang L, Liu J, Li YK, et al. Exploring natural silk protein sericin for regenerative medicine: an injectable, photoluminescent, cell-adhesive 3D hydrogel. Scientific Rep. 2014;4:7064.

10. Kwon JS, Kim SW, Kwon DY, Park SH, Son AR, Kim JH, et al. In vivo osteogenic differentiation of human turbinate mesenchymal stem cells in an injectable in situ-forming hydrogel. Biomaterials. 2014;35:5327-46.

11. Son AR, Kim DY, Park SH, Jang JY, Kim KS, Kim BJ, et al. Direct chemotherapeutic dual drug delivery through intra-articular injection for synergistic enhancement of rheumatoid arthritis treatment. Scientific Reports. 2015;5:14713.

12. Oh NW, Jegal J, Lee KH. Preparation and characterization of nanofiltration composite membranes using polyacrylonitrile (PAN). I. Preparation and modification of PAN supports. J Appl Polym Sci. 2001;80:1854-62.

13. Chaudhary BK, Farrell J. Preparation and characterization of homopolymer polyacrylonitrile-based fibrous sorbents for arsenic removal. Environ Eng Sci. 2014;31:593-601

14. Chen S, Gao H, Chen J, Wu J. Surface modification of polyacrylonitrile fibre by nitrile hydratase from Corynebacterium nitrilophilus. Appl Biochem Biotechnol. 2014:174:2058-66.

15. Chiang YW, Hu CM. Studies of reactions with polymers. VI. The modification of PAN with primary amines. J Polym Sci Part A: Polym Chem. 1990;28:1623-36 
16. Bryjak M, Hodge H, Dach B. Modification of porous polyacrylonitrile membrane. Angew Makromol Chem. 1998;260:25-9.

17. Lohokare HR, Kumbharkar SC, Bhole YS, Kharul UK. Surface modification of polyacrylonitrile based ultrafiltration membrane. J Appl Polym Sci. 2006;101:4378-85

18. Jindal KK, McDougall J, Woods B, Nowakowski L, Goldstein MB. A study of the basic principles determining the performance of several high-flux dialyzers. Am J Kidney Dis. 1989:14:507-11.

19. Chanard J, Lavaud S, Randoux C, Rieu P. New insights in dialysis membrane biocompatibility: relevance of adsorption properties and heparin binding. Nephrol Dial Transpl. 2003;18:252-7.

20. Czop JK, Austen KF. Properties of glycans that activate the human alternative complement pathway and interact with the human monocyte beta-glucan receptor. J Immunol. 1985;135:3388-93.

21. Ramseyer P, Micol LA, Engelhardt EM, Osterheld MC, Hubbel JA, Frey P. In vivo study of an injectabld poly(acrylonitrile)-based hydrogel paste as a bulking agent for the treatment of urinary incontinence. Biomaterials. 2010;31:4613-9.

22. Yeom JS, Hwang B, Yang D, Shin HI, Hahn S. Effect of osteoconductive hyaluronate hydrogels on calvarial bone regeneration. Biomater Res. 2014;18:8

\section{Submit your next manuscript to BioMed Central and take full advantage of:}

- Convenient online submission

- Thorough peer review

- No space constraints or color figure charges

- Immediate publication on acceptance

- Inclusion in PubMed, CAS, Scopus and Google Scholar

- Research which is freely available for redistribution 